\title{
Influence of Intrauterine Maturation on the Pharmacokinetics of Amikacin in the Neonatal Period
}

\author{
B. M. ASSAEL, ${ }^{(30)}$ R. PARINI, F. RUSCONI, AND G. CAVANNA \\ Department of Pediatrics 2 and Newborn Unit, University of Milano Medical School, Milan, Italy
}

\begin{abstract}
Summary
The effect of intrauterine maturation on amikacin disposition was studied in 29 preterm and term neonates. Mean gestational age (weeks) of the patients was $34.5 \pm 3.3$ S.D. and their birth weight $1.980 \pm 920 \mathrm{~g}$.

After the last administration of the drug, amikacin decay was measured in plasma and urine for 100-250 $\mathrm{h}$. The serum concentration versus time profiles were fitted by nonlinear regression analysis. The parameters of a 2- or 3-compartment model with elimination from the central compartment were calculated. Initial elimination $T 1 / 2$, volume of the central compartment, and steady state volume of distribution were significantly related to intrauterine maturation (respectively $r=-0.76 ;-0.63 ;-0.57$ ) whereas no significant linear correlation was found between clearance and gestational age $(r=0.19)$.

Patients with gestational age less than $34 \mathrm{wk}$ had a significantly reduced clearance when compared with the neonates with gestational age greater than $36 \mathrm{wk}(0.78 \pm 0.17$ versus $1.0 \pm 0.4 \mathrm{ml} / \mathrm{h} /$ $\mathrm{kg}, P<0.05)$. The ratio between the volumes of distribution showed that a higher amount of amikacin penetrates the peripheral compartments with increased gestational age. The renal clearance calculated in six patients averaged $66 \%$ of the total body clearance, suggesting that elimination of the drug can occur in the neonate via nonrenal routes. Analysis of the long term urinary elimination of amikacin showed that about $5 \%$ of the total amount of the drug administered in 5-8 days of treatment is retained in the organism. Although quantitatively small, this amount is relevant for the potential nephrotoxicity of the drug.
\end{abstract}

The current recommendations for aminoglycoside antibiotic dosages in children and adults are based upon the recognition that the clinical toxicity of these compounds is associated with elevated serum concentrations but that efficacy too is related to the achievement of concentrations higher than the minimal inhibitory concentrations on the causal bacteria (5). Pharmacokinetic models have been developed in adults and children to improve predictions of serum aminoglycoside concentrations and permit early recognition of patients in whom excessive accumulation is occurring (6, $9,27)$. Several risk factors have been recognized and some of them, such as kidney failure have pharmacokinetic implications.

Clearly, the neonate shows a peculiar situation because rapid changes in body composition and renal function occur early after birth. The different degree of maturation at birth is another influencing factor. Improved intensive care has raised the survival rate of very low birth weight neonates. Hence there is increasing importance in obtaining data on the factors that influence drug disposition in this age group.

The present study was designed to further investigate the pharmacokinetics of amikacin in the neonate. This drug is currently recommended for treatment of infections with gram negative bacteria resistant to other aminoglycosides. Its use in the neonate is still limited. Pharmacokinetic studies on amikacin in the neonate are already available but limited attention has been devoted to the role of intrauterine maturation $(16,22,25,26)$. The present data are part of a larger study concerning the clinical safety of amikacin in the neonatal period.

The effect of the drug on the neonatal kidney and auditory function have been closely monitored and are presented separately (23).

\section{PATIENTS AND METHODS}

Twenty-nine neonates of different gestational ages with confirmed or suspected infections due to gram negative bacteria were given amikacin (Bristol Italiana Sud S.P.A.) at an average dose of $7.2 \mathrm{mg} / \mathrm{kg}$ every $12 \mathrm{hr}( \pm 0.57$ S.D. $)$ The treatment was started during the first or second day of life and stopped when neonates were $7.6 \pm 2.5$ days old.

Gestational age was obtained from obstetric anamnesis and by the scoring method of Finnstrom (10). A good correlation was found between these two methods. The range of gestational ages 28.5-42 wk (mean and S.D., $34.5 \pm 3.3$ ) and of birth weights $0.9-4.5 \mathrm{~kg}(1.98 \pm 0.92)$. According to the study of Gairdner and Pearson (11) $34 \%$ of the neonates were small $(<10$ th percentile), $59 \%$ were adequate, and $7 \%$ were large ( $>97$ th percentile) for their gestational age.

Though concentrations of the drug were monitored during the treatment, on cessation of therapy, the disappearance of the drug was followed in plasma samples taken by heel puncture $(50 \mu \mathrm{l})$ at $30 \mathrm{~min}, 1,2,3,6$, and $12 \mathrm{~h}$ and then daily as long as the concentrations of the drug were measurable. In eight patients, urinary elimination of the drug was followed during the washout period. Samples of urine were collected on spontaneous voiding for periods of 1.5-12 h. A total of 15-23 samples could be collected from six newborns in the 15 days after the end of treatment.

Assay. Serum and urines were kept frozen until analysis. This was performed by a radioimmunoassay (Diagnostic Product Corporation Los Angeles, CA). These assays, commercially available, can be slightly modified to improve their sensitivity. In our hands the assay was reproducible with a lower sensitivity of $0.09 \mu \mathrm{g} / \mathrm{ml}$.

Pharmacokinetic analysis. The advantages of a two-compartment open model in describing serum concentrations versus time profiles of aminoglycoside antibiotics have been well established $(9,14,24,27)$. Such analysis gives better predictability of the serum concentrations after a single dose or multiple treatment, and a more reliable estimate of body clearance and volume of distribution. It reveals the presence of a deep compartment to which the drug binds strongly and which is accounted for by slow decay of the serum and urinary concentrations. Blood sampling in our patients was therefore designed to permit evaluation of the multiexponential decline after cessation of treatment over a period of 150-200 h. Data were fitted by nonlinear regression analysis (20) and the parameters of a two- or three-compartment open model with elimination from the central compartment were calculated. 
Urinary clearance. The urinary excretion rates were plotted against the plasma concentrations at the midpoint of the urinary collection period. When this was not actually measured it was calculated from the nonlinear fit. Renal clearance equals the slope of the straight line resulting from this plot.

The methods followed in the present study were approved by the Committee for Human Experimentation of the Regione Lombardia, according to local legislation.

\section{RESULTS}

At last administration, the predose levels of amikacin were related to the degree of intrauterine maturation as shown in Figure 1. Analysis of the regression between the two variables led to calculation of the following equation, $\mathrm{y}=-0.44 \chi+21$ with a linear correlation coefficient $r=-0.56$. Neonates with gestational age less than 34 wk showed a significantly higher accumulation of the drug.

After cessation of treatment, the decay of the plasma concentration of amikacin followed a 2- or 3-exponential decline (Fig. 2).

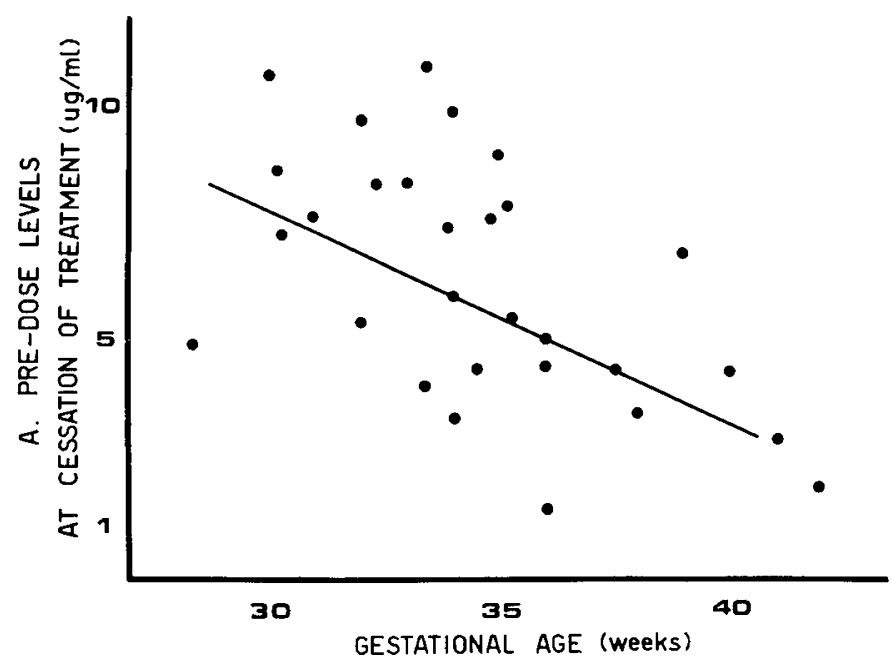

Fig. 1. The figure shows the trough levels of Amikacin $(7.4 \mathrm{mg} / \mathrm{kg}$ every $12 \mathrm{~h}$ for $6-8$ days) at the end of treatment measured in 29 neonates of different gestational age. Equation of the linear regression analysis is given in the text.
Accordingly, the parameters of a 2- or 3-compartment model were calculated and each parameter was related to the gestational ages of the neonates. Mean values are shown in Table 1.

Elimination half-lives (Fig. 3-4). The initial elimination half-life showed a marked decrease in relation to maturation according to the equation T $1 / 2_{\alpha}=-0.66$ wks gestational age (G.A.) $+29(r=$ $-0.76)$. In contrast no correlation was seen between gestational age and the slope of the terminal elimination phase $(\mathrm{T} 1 / 2 \beta=-0.07$ wk G.A. $+65.5 ; r=-0.13$ ) indicating that the slow release of amikacin from the tissue ste res is independent of intrauterine maturation.

Clearance. The relation of total body clearance of amikacin and gestational age is shown in Figure 5. The linear correlation between the two variables is poor $(\mathrm{Clb}=0.007$ wk G.A. +0.72 ; $r=0.19$ ).

Volumes of distribution. The apparent volume of the central compartment and the apparent volume of distribution at steady state were calculated (Fig. 6-7). In both cases an inverse correlation to gestational age was seen. $(\mathrm{Vc}=-0.035 \chi+1.7 ; r=-0.63$; $\mathrm{Vdss}=-0.027 \chi+1.7 ; r=-0.57)$.

To further evaluate whether intrauterine maturation could influence the degree of equilibration of the drug, the individual ratios of $\mathrm{Vc} / \mathrm{Vdss}$ were plotted versus gestational age (Fig. 8) The equation of the linear regression analysis was $\mathrm{Vc} / \mathrm{Vdss}=-0.02 \chi$ +1.28 and $r=-0.55$. This indicates that with increasing gestational age a larger fraction of the drug penetrates the peripheral compartments.

Urinary elimination. The biphasic decay of urinary excretion rate versus time is shown in Figure 2. The elevated amounts of amikacin in the urine permitted better estimation of the terminal phase for intervals at which no drug could be detected in the plasma.

The data of urinary excretion rates versus time measured in

\section{Table 1}

\begin{tabular}{cc} 
Mean $( \pm \mathrm{S} . \mathrm{D}$.$) values of the pharmacokinetic parameters of Amikacin$ \\
\multicolumn{1}{c}{ calculated in 29 premature neonates } \\
\hline $\mathrm{T} 1 / 2_{\alpha}(\mathrm{h})$ & $6.77 \pm 2.87$ \\
$\mathrm{~T} 1 / 2 \beta(\mathrm{h})$ & $62.85 \pm 33.24$ \\
$\mathrm{Vc}(1 / \mathrm{kg})$ & $0.49 \pm 0.18$ \\
$\mathrm{Vdss}(1 / \mathrm{kg})$ & $0.80 \pm 0.23$ \\
$\mathrm{Cl}(\mathrm{ml} / \mathrm{min} / \mathrm{kg})$ & $0.86 \pm 0.29$ \\
\hline
\end{tabular}

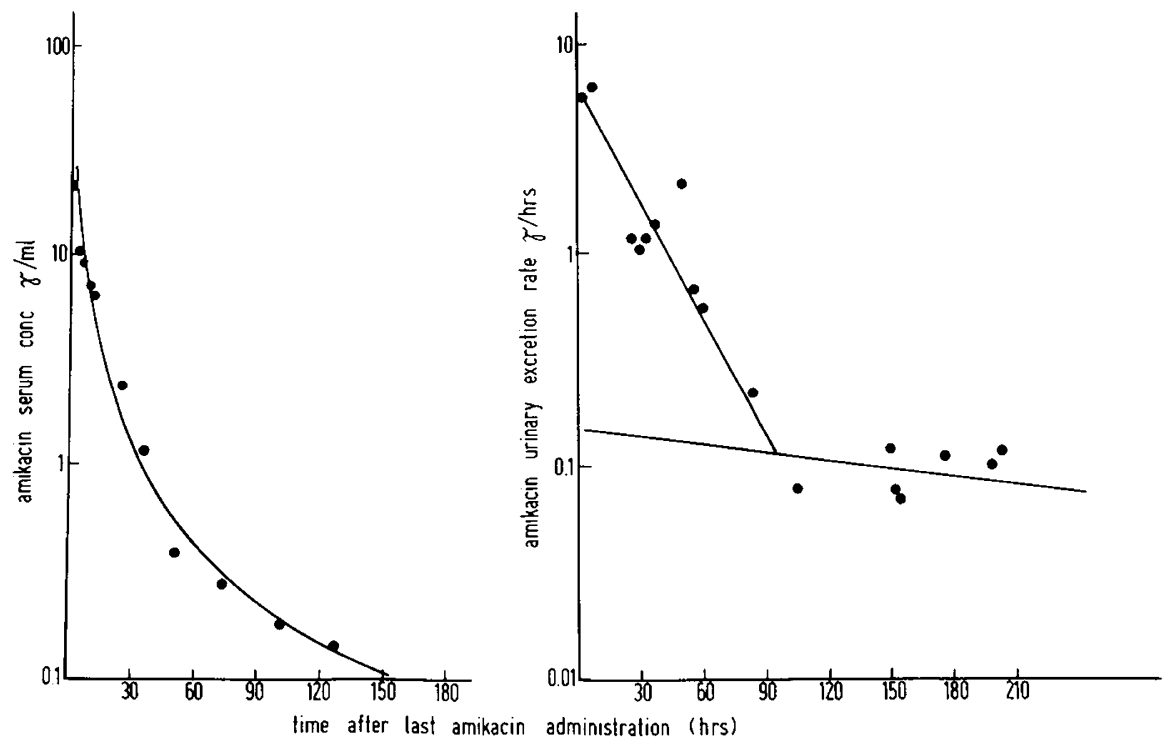

Fig. 2. The figure shows a typical multiexponential decline of Amikacin after cessation of treatment. Serum concentrations are shown on the left and urinary excretion rates on the right. 


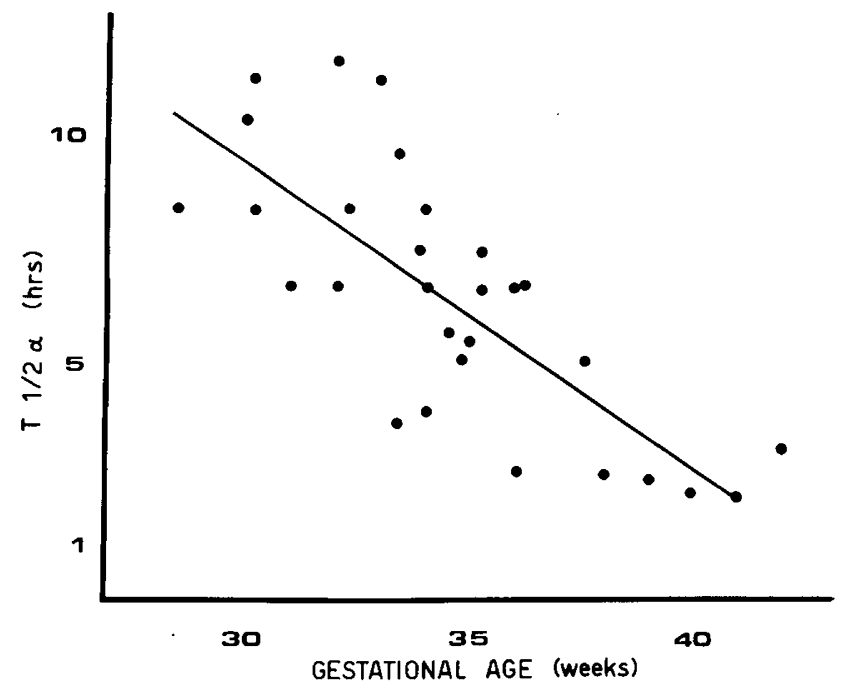

Fig. 3-7. The figures show the relation calculated for the various kinetic parameters of Amikacin and gestational age of 29 premature and term neonates. Symbols and statistical data are given in the text.

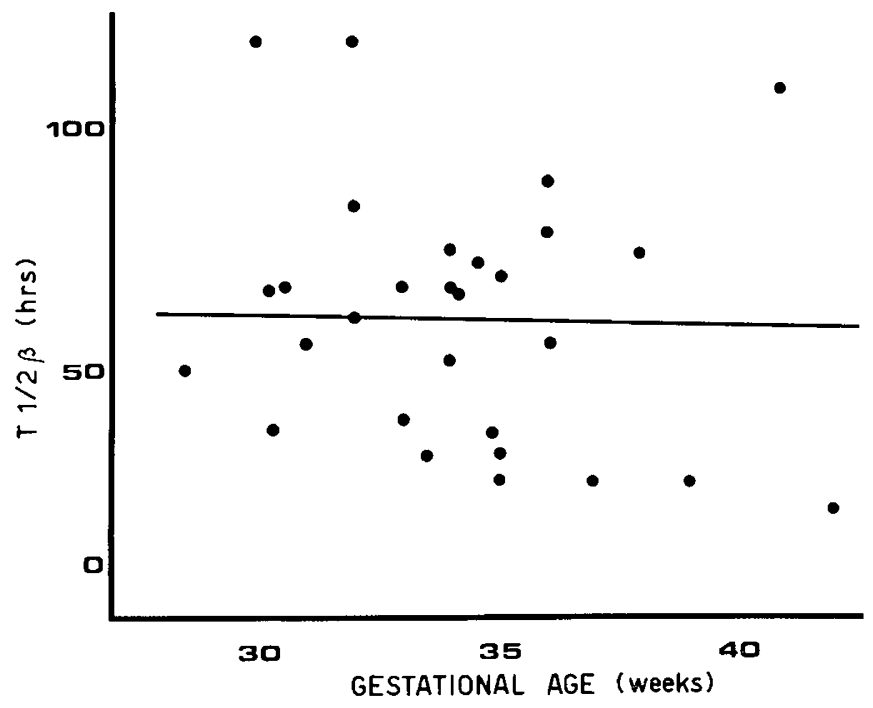

Fig. 4.

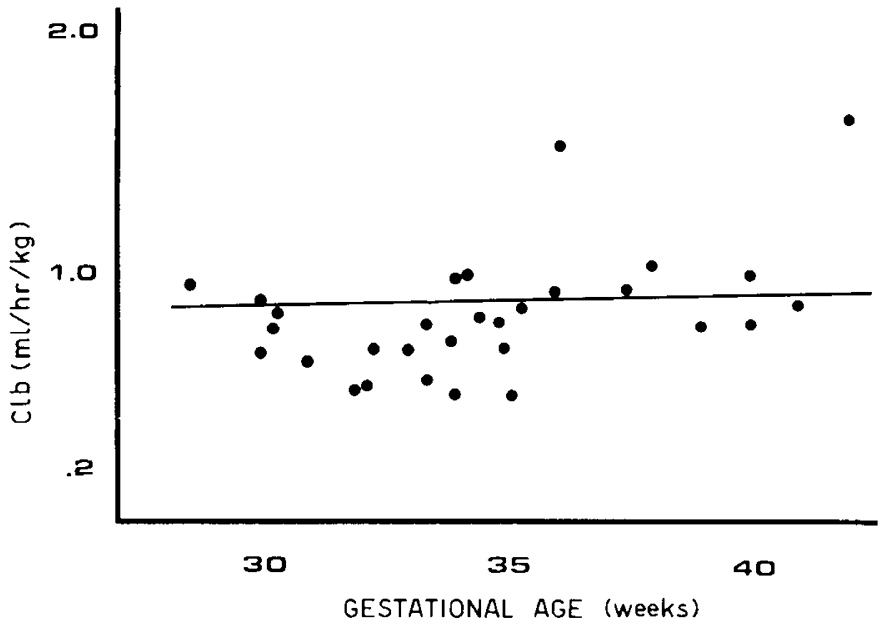

Fig. 5.

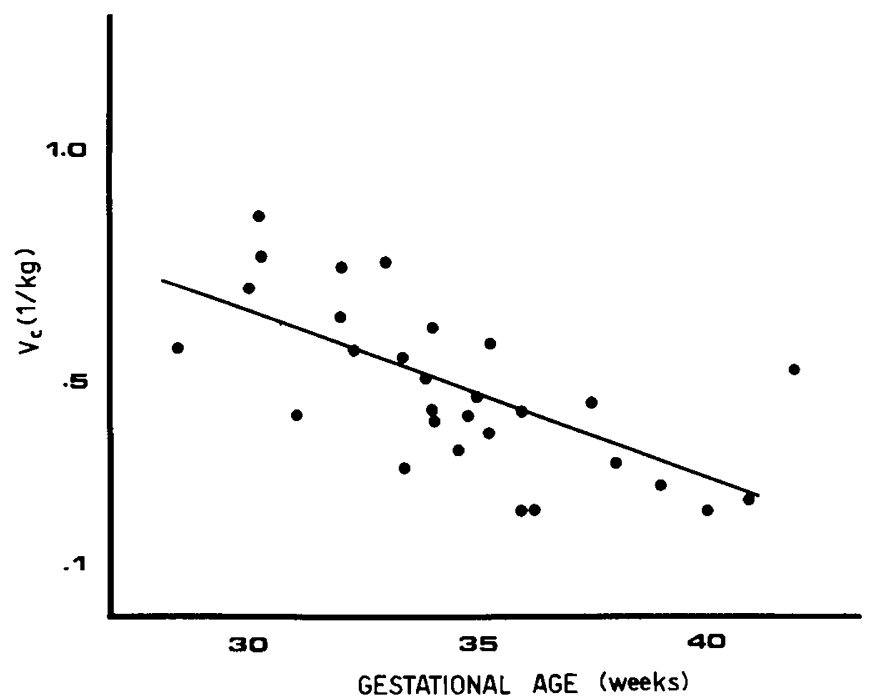

Fig. 6.

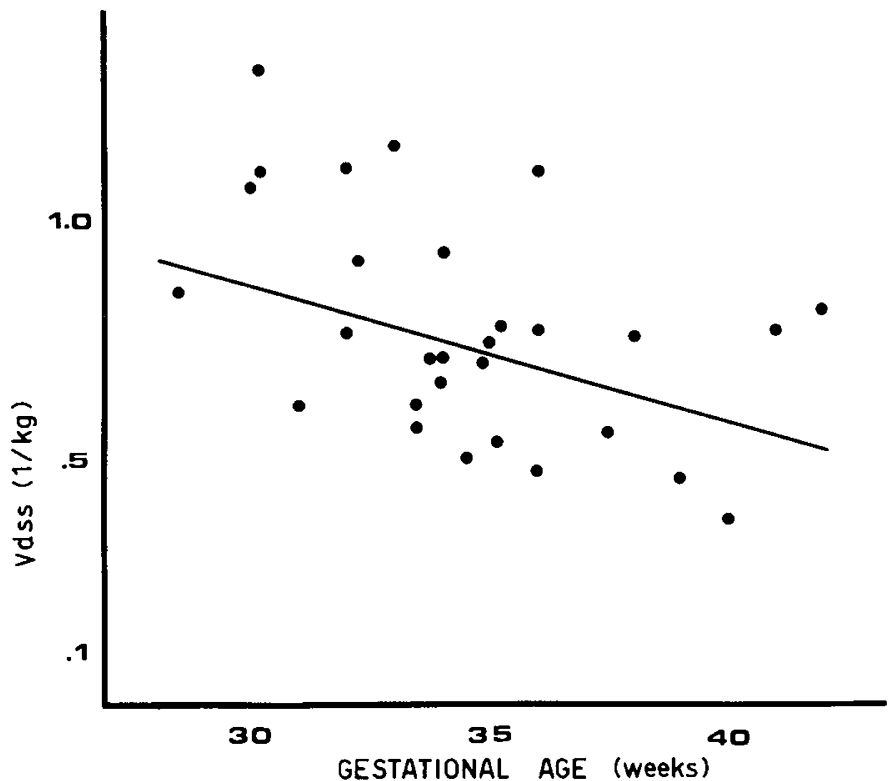

Fig. 7.

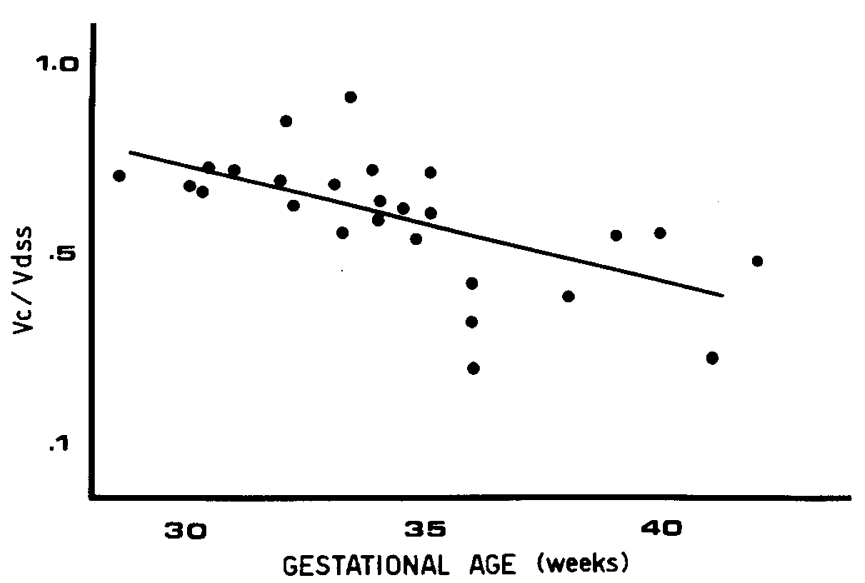

Fig. 8. The figure shows that with increasing gestational age the individual ratios of the central compartment and steady state volume of distribution are decreasing. 


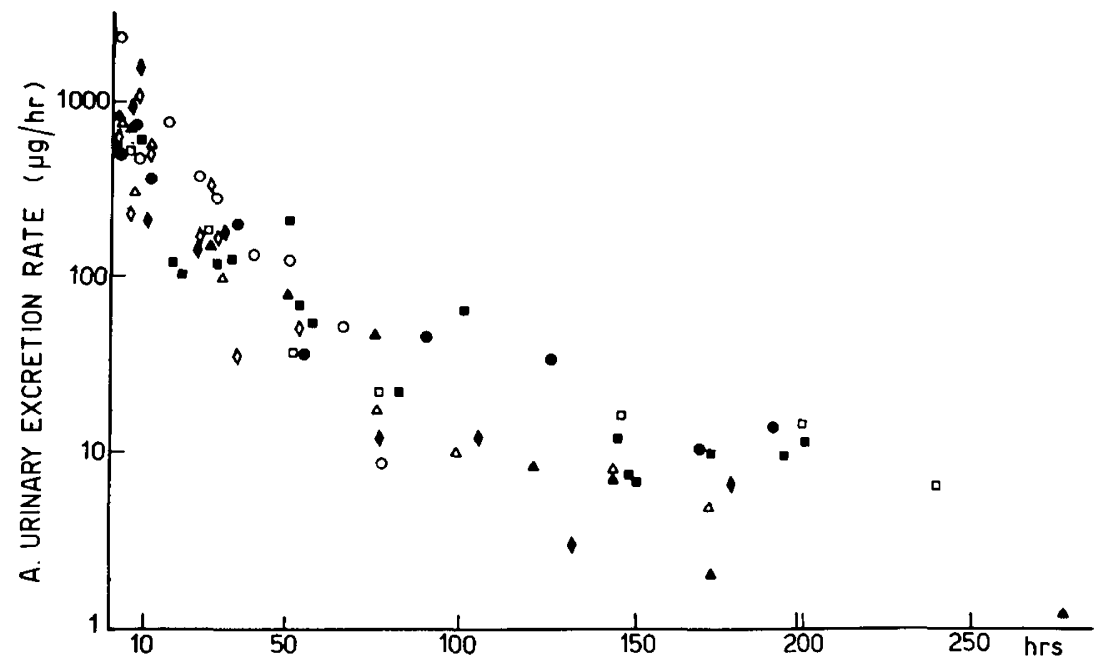

Fig. 9. The figure shows the urinary excretion rates of Amikacin measured in eight patients at various times after cessation of therapy. Each symbol represents the data calculated for a single patient.

Table 2. Urinary clearance of Amikacin and total amount excreted after cessation of therapy in six premature neonates

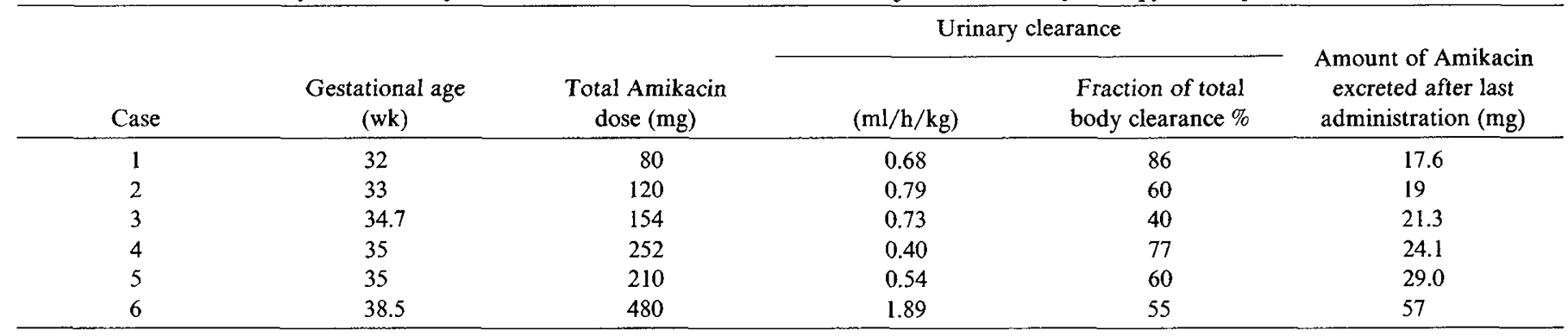

eight neonates are shown in Figure 9. A biphasic decay is seen, which parallels the decay of serum concentrations. The half-life of the terminal phase calculated from the slope of the regression equation of the data of all the patients was $112 \mathrm{~h}$. Sufficient data could be obtained in six patients in order to permit an adequate measurement of the urinary clearance (Table 2). In each case this was lower than the total body clearance; therefore, a substantial amount of amikacin seems to be eliminated by these neonates via nonrenal routes. The total amount of amikacin eliminated in the urine after the last dose was calculated as the area under the curve of the urinary excretion rates versus time extrapolated to infinity. These amounts were linearly correlated to the total dose administered to the infants during treatment $(\mathrm{Y}=0.1 \chi+6 ; r=0.96)$ (Fig. 10). The fraction of the total dose recovered in the urine after the cessation of the treatment was $14.4 \pm 4.3 \%$. The portion under the terminal phase of the urinary excretion versus time curve shown in Figure 9 was $7 \%$ of the total area. This portion represents the amount of the drug retained in the body and slowly released.

\section{DISCUSSION}

Little is known about the factors affecting the toxicity of aminoglycoside antibiotics in the neonate. There is experimental evidence that these effects may be modulated by age: the more immature the animal the lower its sensitivity; however, nephro-or ototoxicity are difficult to detect in the neonate because of methodologic problems. In our patients the possible effects of amikacin on auditory and renal function were monitored. The results of these studies are presented elsewhere (23); however, it can be anticipated that the only detectable significant effect on the kidney was an increase in urinary excretion of the lysosomal enzyme, $\mathrm{N}$ acetyl glucosaminidase.

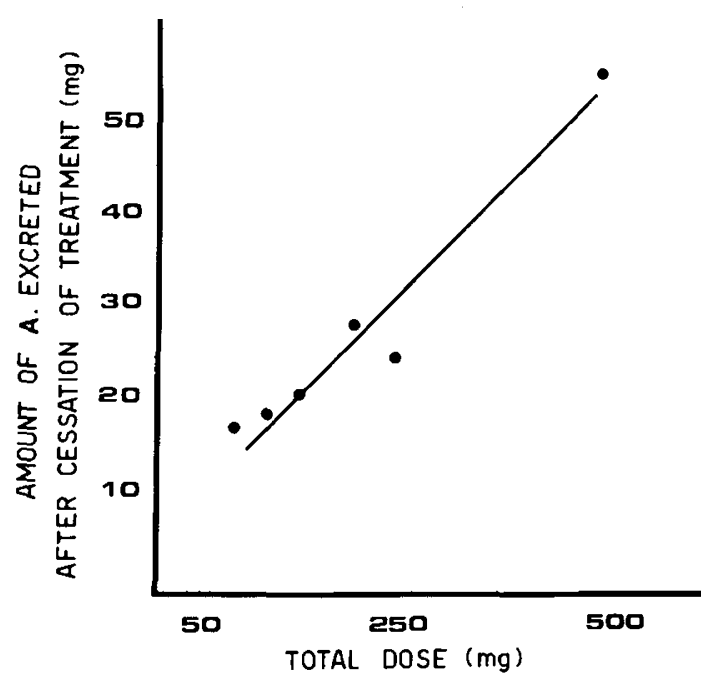

Fig. 10. The figure shows the relationship between the total amount of Amikacin administered during 6-8 days of treatment and the urinary recovery of the drug after cessation of treatment. Statistical analysis is given in the text.

The importance of pharmacokinetics in elucidating some characteristics of aminoglycoside toxicity has been recognized. Because neonates are a far from homogenous group, mainly because of differences in intrauterine maturation we designed our investigation in order to clarify the role of gestational age on the disposition of amikacin. It was hoped that this approach would also offer 
some insight on the toxicologic aspects of these drugs early in development. This was done by examining plasma and urinary disposition of the drug. Analysis of urinary clearance seemed relevant because it can give more information on renal handling of the drug and because urinary concentrations of aminoglycosides are still measurable when the plasma concentrations are below the sensitivity of the analytical methods. This permits more appropriate pharmacokinetic estimate of the characteristics of the drug. Furthermore, measurement of the drug recovered in the urine allows direct calculation of the amount retained in the body. This is preferable to indirect extrapolation from the plasma data.

The pharmacokinetics of amikacin in the neonate has already been studied by other authors. Sardemann et al. (26) studied a group of neonates of different gestational ages and recognized the neonate's low clearance capacity of amikacin and larger volume of distribution compared to adults. Howard and McCracken (16) found longer half-lives of amikacin in neonates with birth weight lower than $2000 \mathrm{~g}$ and in the first 4 days of life than in neonates with higher birth weight and older postnatal age. Myers et al. (22) found that prolonged half-life was related to early gestational age, low birth weight and hypoxemia. These studies therefore already demonstrated the important differences between adults and neonates.

The investigational design of our study permitted us to distinguish the different effects of maturation on the serum and tissue accumulation of aminoglycosides. As expected, serum accumulation of the drug in the first days of life is higher in the very low birth weight neonate ( $<34$ wk G.A.). Little data are available on drug disposition in this age group, which is now receiving more attention. Our data are in accordance with those of Szefler $e t$ al. (29) and of Assael et al. (3) who found similar data for gentamicin. This stresses the importance of longer intervals between doses of aminoglycosides in the very low birth weight neonate. The higher serum accumulation is the consequence of the longer $T 1 / 2_{\alpha}$. No change could be seen in $T 1 / 2 \beta$ as a function of intrauterine maturation.

The decreased $\mathrm{T} 1 / 2_{\alpha}$ with increasing gestational age was not paralleled by an increased clearance of amikacin. This finding merits some discussion in order to avoid an apparent contradiction. Aminoglycosides are primarily eliminated through glomerular filtration and their clearance is well related to the filtration rate (GFR). This is known to increase soon after birth but at different rates in the full term and preterm neonate. Differences in GFR between these two groups may not be evident in the first week of life. Then the full term neonates show a steeper increase in GFR than the preterm and the difference is seen throughout the first weeks of life.

Considered all together, available studies $(1,2,13,28)$ suggest that the differences in GFR between preterm and term neonates are more evident at the extremes of gestational age, i.e., between neonates of less than $34 \mathrm{wk}$ G.A. and full term. Comparison of the clearance calculated in our patients revealed that the group of G.A. less than 34 wk had a mean clearance of $0.78 \mathrm{ml} / \mathrm{h} / \mathrm{kg}$ $( \pm 0.17$ S.D.) and those of G.A. more than $36 \mathrm{wk}$ had a mean clearance of $1.0 \mathrm{ml} / \mathrm{h} / \mathrm{kg}$ ( \pm 0.4 S.D.). The difference between these values is significant at the 0.05 level by the Student's two tails $t$ test. Therefore the pharmacokinetic data can be reconciled with the physiologic pattern of GFR development in premature neonates.

It may be concluded that the decreasing $T 1 / 2 \alpha_{\alpha}$ is also greatly influenced by the concurrent decrease in $\mathrm{Vd}$, related to the reduction in extracellular water occurring with intrauterine maturation. This also stresses the importance of not considering $T 1 / 2$ as a comparative parameter of the capacity to eliminate drugs in premature neonates unless differences in $\mathrm{Vd}$ are carefully excluded.

The relative changes in the $\mathrm{Vc}$ versus Vdss values indicate that not only intrauterine growth causes a change in the total volume in which amikacin is distributed; there is also a change in the degree of equilibration of the drug between the central and the peripheral compartments. Based on the analysis of regression (Fig. 8 ) the fraction of the drug in the central compartment at steady state is $74 \%$ of the total drug in the body in a $28 \mathrm{wk} \mathrm{G.A.} \mathrm{neonate}$ and $47 \%$ in the term newborn. Because aminoglycosides can elicit toxic renal effects and it has been demonstrated that these are related to the degree of accumulation in the peripheral compartment (28), changes in drug distribution are an obvious cause for concern.

The validity of the two-compartment model for predicting tissue accumulation of aminoglycosides has so far been ascertained in adults and might not be applicable in the neonate; however, some recently published experimental data give clear evidence that the renal accumulation of aminoglycosides is an age-dependent phenomenon. Cowan et al. (7) found smaller amounts of gentamicin in puppies than in the adult dog. The same has been found in the rat $(19,21)$. The smaller amount of aminoglycosides found in the kidney of the newborn animal was related in some of these studies to a lower nephrotoxicity.

There is scant data available for the human neonate. In a previous study (4) we found that the changes in the pharmacokinetic characteristics of gentamicin occurring with growth could predict age-related changes in the penetration of the drug in the tissue compartment. To our knowledge direct measurements of aminoglycosides in the human neonatal kidney were reported in only one study (8) where they were found to be lower than the concentration found in adults. This is confirmed by data obtained in our laboratory. A lower accumulation of aminoglycosides in the immature kidney could be explained by a low cortical plasma flow and immature proximal tubular cells $(15,17)$.

Clearly, the role of maturation on the accumulation of aminoglycosides in the human kidney deserves more study and direct evaluation; however, data presented in this study make some contribution in validating the working hypothesis that these compounds might have a different nephrotoxicity according to maturation.

An indirect method of assessing the extent of tissue retention of aminoglycosides would be to measure recovery of the drug in urine over prolonged periods after cessation of the treatment. After a single dose of gentamicin to the adult, Kahlmeter (18) recovered $94 \%$ of the drug in a period of $322 \mathrm{~h}$. Of this, $15 \%$ was retained in the body and slowly excreted during the terminal phase. After multiple doses the fraction of the total retained averaged $5 \%$.

On the other hand in an experimental model Glauser et al. (12) have shown that gentamicin storage might have a protective effect against $E$. coli kidney infection. It is not known whether the prolonged urinary excretion of small and subtherapeutic levels of amikacin might facilitate the emergence of resistance of germs causing urinary tract infections.

These observations prompted us to investigate the urinary kinetics of amikacin in the neonate. The area under the curve extrapolated to infinity obtained by plotting urinary excretion rates versus time gives the total amount eliminated in the urine provided the excretion remains unchanged over the extrapolated portion of the curve. On this assumption, we calculated that an average $14.5 \pm 4.3 \%$ of the total amount of amikacin received in 4-10 days was recovered in the urine of the neonate after the last administration. Of this, $7 \%$ was retained and eliminated in the terminal phase. To our knowledge this is the first attempt to evaluate aminoglycoside retention by the neonate. Interpretation of these findings would require comparison with adults treated with amikacin but no such data are available.

In conclusion, our study shows that the pharmacokinetics of amikacin are profoundly influenced by gestational age and that the kinetic parameters are in accordance with the concepts of developmental physiology. Similarly to the adult, a small \% of the total drug administered is retained in the body. This small portion is of relevance for toxicologic considerations. Maturation not only determines changes in clearance and total volume of distribution but also changes in equilibration between the body compartments. 
Such changes in distribution might be related to the lower toxicity of aminoglycosides demonstrated in the neonate in experimental studies.

\section{REFERENCES AND NOTES}

1. Aperia, A., Brobergen, O., Elinder, G., Herin, P., and Zetterstrom, R.: Postnata development of renal function in pre-term and full-term infants. Acta Pediatr. Scand., 70: 183 (1981)

2. Arant, B. S., Jr.: Developmental patterns of renal functional maturation compared in the human neonate. J. Pediatr., 92: 705 (1978).

3. Assael, B. M., Gianni, V., Marini, A., Peneff, P., and Sereni, F.: Gentamicin dosage in pre-term and term neonates. Arch. Dis. Child., 52: 883 (1977).

4. Assael, B. M., Cavanna, G., Jusko, W. J., Marini, A., Parini, R., Sereni, F., and Viganò, A.: Multiexponential elimination of gentamicin. A kinetic study during development. Dev. Pharmacol. Ther., I: 171 (1980).

5. Barza, M. and Lauerman, M.: Why monitor serum levels of Gentamicin? Clin Pharmacokin., 3: 202 (1978).

6. Colburn, W. A., Schentag, J. J., Jusko, W. J., and Gibaldi, M. A.: A model for the prospective identification of the pre nephrotoxic state during gentamicin therapy. J. Pharmacokin. Biopharm., 6: 179 (1978).

7. Cowan, R. H., Jukkola, A. F., and Arant, B. S., Jr.: Pathophysiologic evidence of gentamicin nephrotoxicity in neonatal puppies. Pediatr. Res., 14: 1204 (1980)

8. Edwards, C. Q., Smith, C. R., Baughman, K. L., Rogers, J. F., and Lietman, P. S.: Concentrations of Gentamicin and Amikacin in human kidneys. Antimicrob. Agents Chemother., 9: 925 (1976).

9. Evans, W. E., Huntley Taylor, R., Feldman, S., Crom, W. R., Rivera, G., and Yee, G. C.: A model for dosing gentamicin in children and adolescents that adjust for tissue accumulation with continuous dosing. Clin. Pharmacokin., 5 . 295 (1980).

10. Finnstrom, O.: Studies on maturity in newborn infants IV. Further observations on the use of external characteristics in estimating gestational age. Acta Pediatr. Scand., 66: 601 (1977).

11. Gairdner, D. and Pearson, J.: A growth chart for premature and other infants. Arch. Dis. Child., 46: 783 (1971)

12. Glauser, M. P., Lyons, J. M., and Braude, A. I.: Prevention of pyelonephritis due to Escherichia coli in rats with gentamicin stored in kidney tissue. J. Infect. Dis., 139: 172 (1979).

13. Guignard, J. P., Torrado, A., DaCunha, O., and Gautier, E.: Glomerular filtration rate in the first three weeks of life. J. Pediatr., 87: 268 (1975)

14. Haughey, D. B., Hilligos, D. M., Grassi, A., and Schentag, J. J.: Two compartments gentamicin pharmacokinetics in premature neonates. A comparison to adults with decreased glomerular filtration rates. J. Pediatr., 96: 325 (1980).

15. Hay, D. A. and Evan, A. P.: Maturation of the proximal tubule in the puppy kidney: a comparison to the adult. Ant. Rec., 195: 273 (1979).
16. Howard, J. B. and McCracken, G. H. Jr.: Pharmacological evaluation of Amikacin in neonates. Antimicrob? Agents Chemother., 8: 56 (1975).

17. Jose, P. A., Logan, A. G., Slotkoff, L. M., Lilienfield, L. S., Calcagno, P. L., and Eisner, G. M.: Intrarenal blood flow distribution in canine puppies. Pediatr. Res., 5: 335 (1971).

18. Kahlmeter, G., Jonsson, S., and Kamme, C.: Longstanding post-therapeutic gentamicin serum and urine concentrations in patients with unimpaired renal function. A pharmacokinetic evaluation. J. Antimicrob. Chemother., 4: 143 (1980).

19. Marre, R., Tarara, N., Louton, T., and Sack, K.: Age-dependent nephrotoxicity and the pharmacokinetics of gentamicin in rats. Eur. J. Pediatr., 133: 25 (1980).

20. Metzler, C. M.: NonLin. A computer program for parameter estimation in nonlinear situations. Technical report n. 7292/7292/005 (The Upjohn Company, Kalamazoo, 1979)

21. Milner, R. D. G., Milner, G. R., and Lancaster, D.: Tissue Gentamicin concentrations in the newborn and adult rat. Pediatr. Res., 13: 161 (1979)

22. Myers, M. G., Roberts, R. J., and Mirhij, N. J.: Effects of gestational age, birth weight, and hypoxemia on pharmacokinetics of Amikacin in serum of infants. Antimicrob. Agents Chemother., 11: 1027 (1977).

23. Parini, R., Rusconi, F., Vigliani, E., Cavanna, G., Cornacchia, L., and Assael, B. M.: Evaluation of the renal and auditory function of neonates treated with Amikacin. Dev. Pharmacol. Ther. in press, (1982).

24. Pechere, J. C. and Dugal, R.: Clinical pharmacokinetics of aminoglycoside antibiotics. Clin. Pharmacokin., 4: 170 (1979)

25. Prober, C. G., Yeager, A. S., and Arvin, A. M.: The effect of chronologic age on the serum concentrations of amikacin in sick term and premature infants. $J$ Pediatr., 98: 636 (1981).

26. Sardemann, H., Colding, H., Hendel, J., Kampam, J. P., Hvidberg, E. F., and Vejlsgaard, R.: Kinetic and dose calculation of Amikacin in the newborn. Clin. Pharmacol. Ther., 20: 59 (1976).

27. Schentag, J. J., Jusko, W. J., Vance, J. W., Cumbo, T. J., Abrutyn, E., Delattre, M., and Gerbracht, L. M.: Gentamicin disposition and tissue accumulation on multiple dosing. J. Pharmacokin. Biopharm., 5: 559 (1977).

28. Siegel, S. R. and Oh, W.: Renal function as ammarker of human fetal maturation. Acta Pediatr. Scand., 65: 481 (1976).

29. Szefler, S. J., Wynn, R. J., Clarke, D. F., Buckwald, S., Shen, D., and Schentag, J. J.: Relationship of gentamicin serum concentrations to gestational age in pre term and term neonates. J. Pediatr., 97: 312 (1980).

30. Request of reprints should be addressed to: B. M. Assael, M.D. Department of Pediatrics. Clinica Pediatrica De Marchi, Via Commenda 9, 20122 Milano, Italy

31. This work was supported by the National Council for Research, Rome, Italy, Grant 80.01162 .83 .115 .4019 . F.R. was supported by a fellowship from the Bristol Italiana Sud S.p. A.

32. Received for publication September 10, 1981.

33. Accepted for publication March 10, 1982. 